\title{
Replaced middle colic artery originating from the coeliac trunk
}

\author{
Geert Maleux • Christel Van Geet
}

Received: 12 March 2010 /Revised: 29 April 2010/Accepted: 30 April 2010/Published online: 11 June 2010

(C) Springer-Verlag 2010

\begin{abstract}
A 12-year-old girl with a medical history of neonatal repair of a lumbosacral meningomyelocoele presented with intermittent lower gastrointestinal haemorrhage. Diagnostic work-up included catheter visceral angiography. Contrast medium injection did not reveal any source of bleeding; however variant vascular anatomy was depicted. Figure 1 shows coeliac angiography with the middle colic artery (small arrows) supplying the transverse colon originated from the coeliac trunk instead of from the superior or inferior mesenteric artery. Overflow of contrast medium into the superior mesenteric artery (arrowheads) and left colic artery (large arrows) is also shown.

This uncommon aberrant anatomy, without further clinical consequences, is only described in cadaver studies at a rate of $0.5-1 \%$ of cases $[1,2]$. However, in cases of negative superior and inferior mesenteric angiography, cannulation of the coeliac trunk may be required to opacify a replaced middle colic artery and a potential bleeding point in the transverse colon.
\end{abstract}

\footnotetext{
G. Maleux $(\bowtie)$

Department of Radiology, University Hospitals Leuven,

Herestraat 49,

3000 Leuven, Belgium

e-mail: geert.maleux@uz.kuleuven.ac.be

C. Van Geet

Department of Pediatrics, University Hospitals Leuven, Leuven, Belgium
}

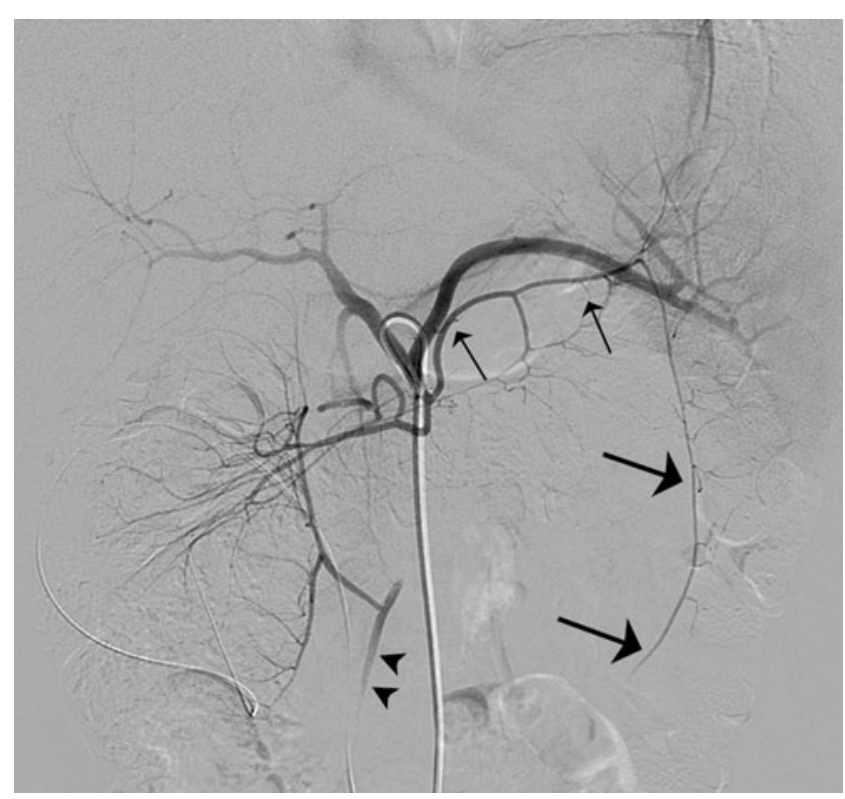

Fig. 1 Coeliac trunk angiography

\section{References}

1. Nelson TM, Pollak R, Jonasson O et al (1998) Anatomic variants of the celiac, superior mesenteric, and inferior mesenteric arteries and their clinical relevance. Clin Anat 1:75-91

2. Yíldírím M, Celik HH, Yíldíz Z et al (2004) The middle colic artery originating from the coeliac trunk. Folia Morphol (Warsz) 63:363-365 